\title{
Agrupamento de modelos de regressão da análise de adaptabilidade e estabilidade de genótipos
}

\author{
Edmar Soares de Vasconcelos( ${ }^{(1)}$, Cosme Damião Cruz $^{(2)}$, Adair José Regazzi(2), Leonardo Lopes Bhering( ${ }^{(3)}$, \\ Tatiana Barbosa Rosado ${ }^{(2)}$ e Fernando Soares de Vasconcelos ${ }^{(4)}$
}

(1)Universidade Estadual do Oeste do Paraná, Departamento de Agronomia, CEP 85960-000 Marechal Cândido Rondon, PR. E-mail: vasconceloses@yahoo.com.br (2)Universidade Federal de Viçosa, Departamento de Biologia Geral, CEP $36571-000$ Viçosa, MG. E-mail: cdcruz@ufv.br, adairreg@ufv.br, tatianarosado@yahoo.com.br ${ }^{(3)}$ Embrapa Agroenergia, Av. W3 Norte (final), PqEb s/no, Edifício Sede, CEP 70770-901 Brasília, DF. E-mail: leobhering@yahoo.com.br (4)Faculdade Integrado de Campo Mourão, Departamento de Agronomia, CEP 87300-970 Campo Mourão, PR. E-mail: fernando.vasconcelos@grupointegrado.br

Resumo - O objetivo deste trabalho foi evidenciar diferenças entre modelos de regressão, obtidos pelo método de Eberhart \& Russell, na análise de adaptabilidade e estabilidade de comportamento de genótipos, e propor uma metodologia de agrupamento dos modelos similares. O teste para verificar a identidade de modelos foi empregado em dados de avaliação de 14 genótipos de milho em oito ambientes. Uma vez rejeitada a hipótese de igualdade dos modelos de regressão, realizou-se o agrupamento desses modelos com base no cálculo do quadrado médio da redução (QMRed) entre pares dos modelos de regressão. Após a obtenção desses valores, foi selecionado o de menor magnitude e verificada sua significância pelo teste F. Na hipótese de esse QMRed não ser significativo, o par de modelos relacionado a ele forma o grupo inicial. O método para verificar a identidade de modelos pode ser usado com sucesso no agrupamento de equações de regressão linear obtidas pelo método de Eberhart \& Russell com o objetivo de estudar a adaptabilidade e a estabilidade de genótipos. O método de agrupamento de modelos similares permite formar grupos de genótipos com o mesmo comportamento estatístico.

Termos para indexação: Zea mays, Eberhart \& Russell, avaliação de genótipos, identidade de modelos.

\section{Regression models grouping for genotype adaptability and stability analysis}

\begin{abstract}
The objective of this work was to provide evidences of differences among regression models, obtained by the Eberhart \& Russell method, for the genotype adaptability and stability analysis, and to propose a methodology for grouping similar models. The test to verify the identity of models was used in the evaluation data of 14 corn genotypes in eight environments. Once the hypothesis of equality of regression models was discarded, model grouping was performed through estimating the mean square of the reduction (MSQRed) between pairs of regression models. After obtaining these values, the smallest one was selected and its significance was verified by the F-test. If this MSQRed is not significant, the model pair related to it comprises the initial group. The models identity verification method can be successfully used for grouping linear regression equations, obtained by the Eberhart \& Russell method, in order to study genotype adaptability and stability. The similar models grouping allow the formation of genotype groups with the same statistical behavior.
\end{abstract}

Index terms: Zea mays, Eberhart \& Russell, genotype evaluation, identity of models.

\section{Introdução}

A avaliação do desempenho de genótipos em diferentes ambientes é comum em programas de melhoramento, pois permite estabelecer o comportamento dos genótipos em relação às variações ambientais (Carvalho et al., 2002).

Para a avaliação do comportamento dos genótipos, diferentes métodos de análise de adaptabilidade são utilizados, entre os quais destaca-se o método de Eberhart \& Russell (1966), que baseia-se em análise de regressão linear simples de uma variável dependente em relação a um índice ambiental (Cruz et al., 2004).

Graybill (1976) apresentou um método para verificar a identidade de $\mathrm{H}$ modelos de regressão linear simples, empregando o teste F. Regazzi (1993) utilizou essa metodologia, tendo considerado o ajustamento de dados de observação relativos a $\mathrm{H}$ equações de regressão polinomial do segundo grau, com auxílio da técnica dos polinômios ortogonais. No decorrer desse processo, esse mesmo autor concluiu que a identidade de modelos de regressão linear simples, ou múltipla, 
e a igualdade de qualquer subconjunto de parâmetros podem ser verificadas por meio do teste F. Essa metodologia foi apresentada por Regazzi (1996) que, nesse trabalho, avaliou o ajustamento de $\mathrm{H}$ equações de regressão no caso de justaposição de submodelos polinomiais.

Regazzi (1999) também descreveu em detalhes o teste para verificar as hipóteses avaliadas por Regazzi (1993), e dessa vez considerou delineamentos experimentais, configurados em dados com repetições. Trabalhos de avaliação do ganho de peso em bovinos (Véras et al., 2001), do conteúdo corporal em novilhos (Zervoudakis et al., 2002) e das exigências nutricionais de macronutrientes para novilhos (Leonel et al., 2006) usaram o método de identidade de modelos para comparação das equações obtidas em cada caso.

As equações de regressão podem ser comparadas por outros métodos, além do apresentado por Regazzi, tais como: os métodos das variáveis Dummy (binárias), da análise de variância, bem como o método das comparações múltiplas. Magalhães et al. (2004) verificaram que os métodos de identidade de modelos das variáveis Dummy e da análise de variância proporcionam resultados similares na comparação de equações de regressões.

Os modelos de regressão ajustados para o estudo de adaptabilidade e estabilidade, no entanto, não são submetidos a testes estatísticos para diferenciá-los.

O objetivo deste trabalho foi evidenciar a existência de diferença entre modelos de regressão na análise de adaptabilidade e estabilidade do comportamento de genótipos, obtidos pelo método proposto por Eberhart \& Russell (1966), e apresentar uma metodologia de agrupamento dos modelos de regressão similares.

\section{Material e Métodos}

O teste para verificar a identidade de modelos, descrito por Regazzi (1993, 1996), foi empregado sobre as equações de regressão obtidas com base na metodologia de Eberhart \& Russell (1966). Os H modelos de regressão são dados por:

$$
\begin{gathered}
Y_{1 j}=\beta_{0_{1}}+\beta_{1_{1}} I_{j}+\varepsilon_{1 j}, Y_{2 j}=\beta_{0_{2}}+\beta_{1_{2}} I_{j}+\varepsilon_{2 j}, \ldots, \\
Y_{H j}=\beta_{0_{H}}+\beta_{1_{H}} I_{j}+\varepsilon_{H j},
\end{gathered}
$$

em que, $Y_{\text {hj }}$ é a j-ésima observação do h-ésimo modelo, sendo $\mathrm{j}=1,2, \ldots, \mathrm{m}$, em que $\mathrm{m}$ o é número de ambientes, e $\mathrm{h}=1,2, \ldots, \mathrm{H}$, em que $\mathrm{H}$ é o número de modelos; $\beta_{0 \mathrm{~h}}$ é o valor da média do genótipo do h-ésimo modelo; $\beta_{1 \mathrm{~h}}$ é o valor do coeficiente de regressão do h-ésimo modelo; $I_{j}$ é o valor do índice ambiental,

$$
I_{j}=\frac{1}{g} \sum_{h=1}^{g} Y_{h j}-\frac{1}{m g} Y_{. .}
$$

em que g é o número de genótipos $(\mathrm{g}=\mathrm{H}), \mathrm{Y}_{\mathrm{hj}} \mathrm{o}$ valor relativo à observação do h-ésimo genótipo no j-ésimo ambiente, e

$$
\sum_{j=1}^{m} I_{j}=0
$$

$\varepsilon_{\mathrm{hj}}$ é o erro aleatório do h-ésimo modelo e j-ésimo ambiente, supostamente independente e normalmente distribuído, com média zero e variância constante, $\sigma^{2}$, $\varepsilon_{\mathrm{hj}} \sim \operatorname{NID}\left(0, \sigma^{2}\right)$.

A hipótese nula testada foi: $\mathrm{H}_{0}: \beta_{1}=\beta_{2}=\ldots=\beta_{\mathrm{H}}$ (os $\mathrm{H}$ modelos são idênticos) vs. $\mathrm{H}_{\mathrm{a}}: \beta_{\mathrm{h}} \neq \beta_{\mathrm{h}^{\prime}}$ para pelo menos um $\mathrm{h} \neq \mathrm{h}$ ' (pelo menos um modelo difere dos demais). Neste caso de regressão linear simples, tem-se que $\beta_{\mathrm{h}}=\left[\beta_{\text {oh }} \beta_{\mathrm{lh}}\right]^{\prime}$, com $\mathrm{h}=1,2, \ldots, \mathrm{H}$, de maneira que o h-ésimo modelo pode ser escrito por: $\mathrm{Y}_{\mathrm{h}}=\mathrm{X}_{\mathrm{h}} \beta_{\mathrm{h}}+\varepsilon_{\mathrm{h}}$, em que, $\mathrm{Y}_{\mathrm{h}}$ é um vetor $\mathrm{m} \times 1$ de observações, sendo $\mathrm{m}$ o número de ambientes; $\mathrm{X}_{\mathrm{h}}$ é uma matriz $\mathrm{m} \times \mathrm{p}$ de constantes conhecidas, sendo $\mathrm{p}$ o número de parâmetros, com valor igual a 2, uma vez que tem-se apenas $\beta_{0 \mathrm{~h}}$ e $\beta_{1 \mathrm{~h}} ; \beta_{\mathrm{h}}$, é um vetor de p x $1(\mathrm{p}=2)$ de parâmetros obtidos na análise de Eberhart \& Russell (1966); $\varepsilon_{\mathrm{h}}$ é um vetor $\mathrm{m} \times 1$ de erros aleatórios, $\operatorname{NID}\left(\Phi, \sigma^{2}\right)$. Esses $\mathrm{H}$ modelos podem ser escritos na forma do modelo linear geral $\mathrm{Y}=\mathrm{X} \beta+\varepsilon$, conforme apresentado por Regazzi (1993, 1996). O modelo estatístico adotado pressupõe homogeneidade das $\mathrm{H}$ variâncias residuais, tendo-se obtido, assim, um estimador comum de variância residual, cuja estimativa é utilizada como denominador da estatística $\mathrm{F}$ de Snedecor. A violação dessa pressuposição, em casos extremos, pode levar a erros nas conclusões. Sua verificação pode ser feita com o auxílio de um dos testes de homogeneidade de variâncias ou seguindo-se um critério prático. De acordo com Gomes (1990), quando a relação entre a maior e a menor variância é inferior a sete, é quase sempre possível combinar as variâncias residuais e obter uma estimativa comum. Quando esse quociente for além de sete, é conveniente que se considere, separadamente, subgrupos de modelos nos quais se tenha dentro de cada subgrupo, uma 
razoável homogeneidade de variâncias. Neste estudo, o valor da relação entre o maior e o menor quadrado médio residual foi inferior a sete, tendo-se admitido a homogeneidade das variâncias residuais.

A soma de quadrados de parâmetros, relativa ao modelo completo (SQPc), é dada por: $\mathrm{SQPc}=\beta^{\prime} \mathrm{X}^{\prime} \mathrm{Y}$, com $2 \mathrm{H}$ graus de liberdade. A soma de quadrados total não corrigida (SQTot) é dada por: $S Q T o t=Y^{\prime} Y$, com $\mathrm{Hm}$ graus de liberdade. A soma de quadrados do resíduo (SQRes) é obtida por:

$\mathrm{SQRes}=\mathrm{SQTot}-\mathrm{SQPc}=\mathrm{Y}^{\prime} \mathrm{Y}-\beta^{\prime} \mathrm{X}^{\prime} \mathrm{Y} ; \mathrm{SQRes}=\mathrm{SQRes}(\mathrm{h})$

com $\mathrm{Hm}$ - 2H graus de liberdade.

Sob a hipótese de nulidade, tem-se que $\beta_{1}=\beta_{2}=\ldots=\beta_{\mathrm{H}}$, o que gera o modelo reduzido, ou seja, $\mathrm{H}$ modelos idênticos. Dessa forma, pode-se escrever $\mathrm{Y}_{\mathrm{ij}}=\theta_{0}+\theta_{1} \mathrm{I}_{\mathrm{j}}+\varepsilon_{\mathrm{ij}}$, em que, $\theta_{0}$ e $\theta_{1}$ são os dois parâmetros comuns, que nessa hipótese representaria todos os modelos. Em notação matricial tem-se $\mathrm{Y}=\mathrm{Z} \theta+\varepsilon$, em que, $Y$ é o vetor dos valores observados da variável dependente, de dimensão $\mathrm{Hm} \times 1$; Z é uma matriz $\mathrm{Hm}$ x 2 de constantes conhecidas; $\theta$ é um vetor 2 x 1 de parâmetros $\left(\theta_{0}\right.$ e $\left.\theta_{1}\right)$; $\varepsilon$ é um vetor de erros aleatórios de dimensão $\mathrm{Hm}$ x 1.

O sistema de equações normais relativo ao modelo reduzido, obtido pelo método dos mínimos quadrados ordinários, propicia uma estimativa para os valores do vetor $\theta$ (estimativas de $\theta_{0}$ e $\theta_{1}$ ), dada por: $\hat{\theta}=\left(Z^{\prime} Z\right)-1 Z^{\prime} Y$. A soma de quadrados de parâmetros relativo ao modelo reduzido (SQPr) é dada por: $\mathrm{SQPr}=\theta^{\prime} \mathrm{Z}^{\prime} \mathrm{Y}$, com dois graus de liberdade.

A diferença entre a SQPc e a SQPr gera a soma de quadrados da redução (SQRed), ou seja, a redução que $\mathrm{H}_{0}$ provoca na soma de quadrados de parâmetros do modelo completo, a qual propicia o teste estatístico que tem como denominador o quadrado médio do resíduo do modelo completo, de modo que: SQRed = SQPc - SQPr, com 2(H - 1) graus de liberdade.

$\mathrm{O}$ valor da estatística $\mathrm{F}\left(\mathrm{F}_{\text {calc }}\right)$, é obtido por: $\mathrm{F}_{\text {calc }}=[(\mathrm{SQPc}-\mathrm{SQPr}) / 2(\mathrm{H}-1)] /[\mathrm{SQRes} /(\mathrm{Hm}-2 \mathrm{H})]$.

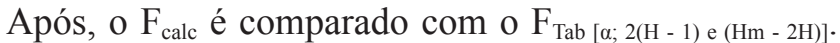
Rejeita-se a hipótese nula $\mathrm{H}_{0}$ se $\mathrm{F}_{\text {calc }} \geq \mathrm{F}_{\text {Tab. }}$ A não rejeição de $\mathrm{H}_{0}$ implica que os $\mathrm{H}$ modelos não diferem estatisticamente. Assim a equação ajustada com as estimativas dos parâmetros comuns pode ser usada como uma estimativa das $\mathrm{H}$ equações envolvidas. Contudo, a rejeição de $\mathrm{H}_{0}$ implica na existência de pelo menos um dos genótipos com comportamento diferente dos demais, o que exige o agrupamento dos genótipos que apresentam comportamentos similares. Para facilitar o entendimento, o esquema da análise de variância é apresentado na Tabela 1.

Para agrupar os modelos, realiza-se o cálculo da SQRed entre todos os pares de modelos de regressão. O procedimento é semelhante ao apresentado para a análise que envolve todos os modelos, descrita anteriormente, porém agora serão envolvidos apenas dois modelos em cada análise. Esse procedimento originará $(\mathrm{H}(\mathrm{H}-1)) / 2$ resultados de SQRed, um valor para cada par de modelos distinto.

A hipótese nula a ser testada foi: $\mathrm{H}_{0}: \beta_{\mathrm{i}}=\beta_{\mathrm{i}^{\prime}}$ (os modelos são idênticos) vs. $\mathrm{H}_{\mathrm{a}}: \beta_{\mathrm{i}} \neq \beta_{\mathrm{i}^{\prime}}$ (o modelo i difere do modelo i'). Calcula-se SQRed para todos os possíveis pares de modelos, seleciona-se o de menor magnitude e verifica-se sua significância pelo teste $\mathrm{F}$, sendo: $\mathrm{F}_{\text {calc }}=[(\mathrm{SQRed}) / 2] /[\mathrm{SQRes} /(\mathrm{Hm}-2 \mathrm{H})]$

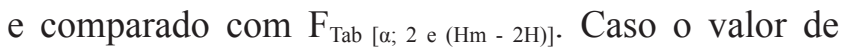
$F_{\text {calc }}$ seja estatisticamente significativo, tem-se que os modelos apresentam comportamentos distintos entre si, contudo se o valor de $F_{\text {calc }}$ for não significativo, o par de genótipos que proporcionou esse $\mathrm{F}_{\text {calc }}$ originará o primeiro grupo, no qual as equações de regressão são estatisticamente idênticas.

A partir do estabelecimento do grupo inicial, testa-se a inclusão de um novo modelo ao grupo preestabelecido. Para tanto é necessário uma nova análise envolvendo os dois modelos agrupados com um terceiro modelo, ainda não agrupado, o que proporciona um novo sistema, agora com três equações de regressão. $\mathrm{O}$ procedimento é semelhante ao apresentado para

Tabela 1. Esquema da análise de variância relativa ao teste da hipótese nula $\mathrm{H}_{0}$ : $\beta_{1}=\beta_{2}=\ldots=\beta_{\mathrm{H}}$ (os $\mathrm{H}$ modelos são idênticos).

\begin{tabular}{lcccc}
\hline Fonte de variação & $\mathrm{GL}$ & Soma de quadrados & Quadrado médio & \\
\hline Parâmetro (completo) & $2 \mathrm{H}$ & $\mathrm{Q}_{1}=\hat{\beta}^{\prime} \mathrm{X}^{\prime} \mathrm{Y}$ & \\
Parâmetro (reduzido) & 2 & $\mathrm{Q}_{2}=\hat{\theta}^{\prime} \mathrm{X}^{\prime} \mathrm{Y}$ & $\mathrm{V}_{1}=\mathrm{Q}_{3} /[2(\mathrm{H}-1)]$ & $\mathrm{V}_{2}=\mathrm{Q}_{4} /(\mathrm{Hm}-2 \mathrm{H})$ \\
Redução $\left(\mathrm{H}_{0}\right)$ & $2(\mathrm{H}-1)$ & $\mathrm{Q}_{3}=\mathrm{Q}_{1}-\mathrm{Q}_{2}$ & \\
Resíduo & $\mathrm{Hm}-2 \mathrm{H}$ & $\mathrm{Q}_{4}=\mathrm{Q}_{5}-\mathrm{Q}_{1}$ & \\
\hline Total & $\mathrm{Hm}$ & $\mathrm{Q}_{5}=\mathrm{Y}^{\prime} \mathrm{Y}$ & \\
\hline
\end{tabular}


a análise que envolve todos os modelos, descrita anteriormente, porém agora serão envolvidos os dois modelos agrupados inicialmente, com cada um dos modelos ainda não agrupados. Esse procedimento irá originar $\mathrm{H}$ - 2 resultados de SQRed.

A hipótese nula a ser testada é: $\mathrm{H}_{0} \beta_{\mathrm{i}}=\beta_{\mathrm{i}}$, $=\beta_{\mathrm{i}}$, (os modelos são idênticos) vs. $\mathrm{H}_{\mathrm{a}}: \beta_{\mathrm{i}}=\beta_{\mathrm{i}^{\prime}} \neq \beta_{\mathrm{i}}$ " (o modelo $\mathrm{i}$ " não é idêntico aos modelos i e i').

Calcula-se SQRed nos casos possíveis e novamente verifica-se a significância do menor valor, pelo teste $\mathrm{F}$, sendo: $\mathrm{F}_{\text {calciii" }}=[(\mathrm{SQRed} / 4)] /[\mathrm{SQRes} /(\mathrm{Hm}-2 \mathrm{H})]$, e

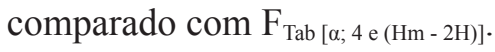

Se o teste F for não significativo, então o genótipo i" será integrado ao grupo inicial, e fará parte dos genótipos com padrão de respostas similares. Após a inclusão de um novo genótipo no grupo, deverá se tentar incluir outro, e esse ciclo será repetido até que o menor valor da SQRed resulte em um $\mathrm{F}_{\text {calc }}$ estatisticamente significativo, que indicaria que os grupos de genótipos e os genótipos não agrupados possuem modelos de regressão diferentes entre si.

Depois de estabelecido o primeiro grupo com genótipos cujas equações de regressão não diferem estatisticamente, pode-se iniciar uma nova tentativa de agrupamento com os modelos ainda não agrupados (Figura 1). Para exemplificar o uso desse método, foi avaliado o comportamento de 14 genótipos de milho em oito ambientes, cujos resultados foram retirados de Costa (1995) (Tabela 2).

As análises de adaptabilidade e estabilidade e também de identidade de modelos de regressão foram

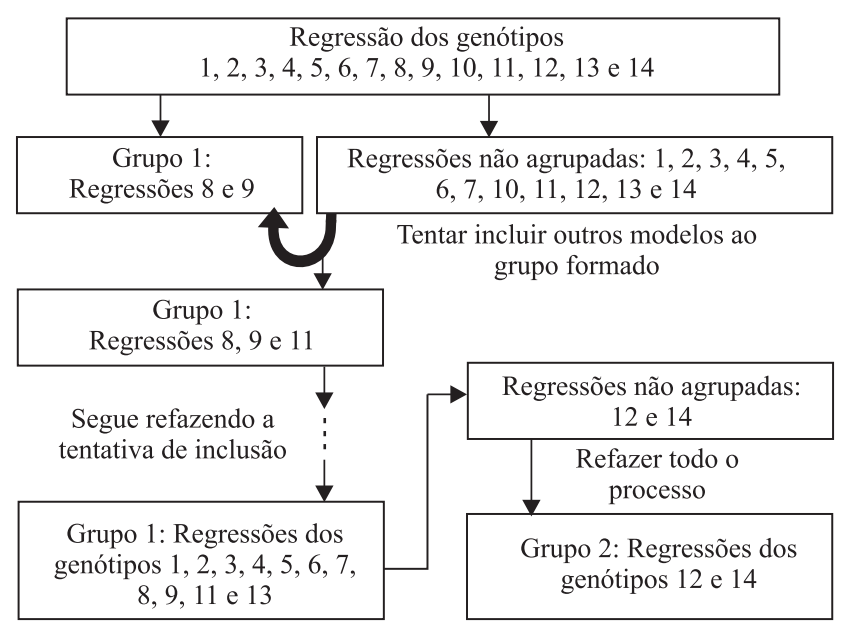

Figura 1. Sequência esquemática do agrupamento dos modelos de regressão de adaptabilidade e estabilidade. realizadas com o auxílio do aplicativo computacional Genes (Cruz, 2006).

\section{Resultados e Discussão}

$\mathrm{Na}$ verificação da relação entre o maior e o menor quadrado médio do resíduo de cada regressão (Tabela 2), obteve-se o valor 6,9607, inferior a sete, conforme sugerido por Gomes (1990), o que permitiu a análise de todos os modelos, simultaneamente. Vale ainda ressaltar que os erros experimentais apresentaram normalidade, o que possibilitou a inferência estatística utilizando-se a distribuição F.

Na Tabela 3 estão descritos os resultados da análise de variância que testam a hipótese de todos os modelos de regressão serem idênticos. Como o valor de $F_{\text {calc }}$ foi maior que o de $\mathrm{F}_{\mathrm{Tab}}$, pode-se afirmar que pelo menos um modelo difere estatisticamente dos demais, pelo teste F.

Com a finalidade de discriminar os genótipos com diferentes comportamentos, foram obtidos os valores do QMRed para cada par de regressão (Tabela 4). Os genótipos 8 e 9 apresentaram o menor valor de QMRed (0,0223), que resultou no $\mathrm{F}_{\text {calc }}=0,05$, com 2 e 84 graus de liberdade, não significativo a $1 \%$ pelo teste F. Esse resultado evidencia que esses genótipos apresentam comportamento idêntico e, portanto, passam a constituir o grupo inicial (Figura 1).

Tabela 2. Estimativas de quadrados médios do resíduo (QMR) de cada regressão, de parâmetros de adaptabilidade e estabilidade do caráter produtividade de espigas de milho em Mg ha ${ }^{-1}$, segundo a metodologia de Eberhart \& Russell (1966), além dos valores de $\mathrm{R}^{2}$ de cada modelo.

\begin{tabular}{lcccc}
\hline Tratamento & QMRregressão & Média $\left(\hat{\beta}_{0}\right)$ & $\hat{\beta}_{1}$ & $\mathrm{R}^{2}(\%)$ \\
\hline 1 & 0,7824 & 4,44 & 0,9034 & 89,32 \\
2 & 0,2593 & 4,76 & 1,1225 & 97,49 \\
3 & 0,4237 & 4,39 & 0,9478 & 93,75 \\
4 & 0,3263 & 4,58 & 0,8544 & 94,29 \\
5 & 0,3587 & 4,99 & 1,0280 & 95,65 \\
6 & 0,6786 & 4,08 & 1,0814 & 92,96 \\
7 & 0,3668 & 4,29 & 1,0209 & 97,36 \\
8 & 0,2904 & 4,63 & 1,0220 & 96,04 \\
9 & 0,3381 & 4,56 & 0,9911 & 95,46 \\
10 & 0,5062 & 5,00 & 1,0808 & 95,27 \\
11 & 0,1146 & 4,76 & 1,0556 & 98,56 \\
12 & 0,4800 & 5,84 & 1,1190 & 95,95 \\
13 & 0,4089 & 5,10 & 0,8988 & 94,39 \\
14 & 0,7977 & 5,60 & 0,8743 & 88,94 \\
\hline
\end{tabular}

Fonte: Costa (1995). 
$\mathrm{Na}$ tentativa de inclusão de um novo genótipo ao grupo inicial, obtiveram-se os valores da QMRed relativos aos genótipos do grupo inicial, com cada um dos genótipos ainda não agrupados (Tabela 4). O menor valor da QMRed $(0,0652)$ foi obtido entre os genótipos 8,9 e 11 , que não foi significativo a $1 \%$ de probabilidade pelo teste $\mathrm{F}$, de maneira que o genótipo 11 passa também a integrar o grupo inicial.

Repetiram-se as análises descritas anteriormente, até que os modelos $1,2,3,4,5,6,7,8,9,10,11$ e 13 fossem agrupados. Na tentativa de inclusão de outro modelo ao grupo, obtiveram-se os quadrados médios da redução descrito na Tabela 4. Como o menor quadrado médio da redução foi significativo a 5\% pelo

Tabela 3. Análise de variância gerada para testar a identidade de modelos de regressão obtidos pelo método de Eberhart \& Russell (1966).

\begin{tabular}{lcccc}
\hline FV & GL & Soma de quadrados & Quadrado médio & $\mathrm{F}_{\text {calculado }}$ \\
\hline Parâmetro (c) & 28 & $3.260,7217$ & $1.16,4543$ & \\
Parâmetro (r) & 2 & $3.230,8906$ & $1.615,4453$ & \\
Redução $\left(\mathrm{H}_{0}\right)$ & 26 & 29,8311 & 1,1474 & $2,62^{* *}$ \\
Resíduo & 84 & 36,7920 & 0,4380 & \\
\hline Total & 112 & $3.297,5137$ & & \\
\hline
\end{tabular}

**Significativo a $1 \%$ de probabilidade, pelo teste $\mathrm{F}$. teste $\mathrm{F}$, considerou-se que o grupo 1 estava completo com os genótipos já agrupados, e realizou-se nova análise para possível agrupamento dos modelos ainda não agrupados. A partir dessa análise, um novo grupo foi obtido com as regressões dos genótipos 12 e 14 .

Os genótipos 1, 2, 3, 4, 5, 6, 7, 8, 9, 10, 11 e 13 podem ser representados por um valor comum de beta zero e de beta um (4,63 e 1,0006, respectivamente), e apresentam um coeficiente de determinação $\left(\mathrm{R}^{2}\right)$ igual a $98,46 \%$.

Os genótipos 12 e 14 também puderam ser representados por um valor comum de beta zero e de beta um (5,72 e 0,9967, respectivamente), e apresentaram um $\mathrm{R}^{2}$ igual a $98,51 \%$. Portanto, apenas duas equações comuns explicaram as quatorze equações de regressão inicialmente estabelecidas.

Os valores de beta zero da regressão originada a partir da produção dos genótipos 12 e 14 possuem maior magnitude que dos demais genótipos. Esses valores estão associados à condição de estabilidade de produção, que, neste caso, foi detectada pelo método de agrupamento de modelos. Como os genótipos 12 e 14 alcançaram valores de beta um próximo ao dos demais genótipos, acredita-se que, nesse caso, os valores de beta zero (estabilidade de produção) foram determinantes na diferenciação dos modelos de regressão.

Tabela 4. Estimativas dos quadrados médios da redução (QMRed) dos pares de regressões avaliadas, entre os modelos 8, 9 e os demais ainda não agrupados, e também entre os modelos $1,2,3,4,5,6,7,8,9$, 10, 11 e 13 e os modelos 12 e 14 , na tentativa de acrescentar um novo modelo ao grupo inicial.

\begin{tabular}{|c|c|c|c|c|c|c|c|c|c|c|c|c|c|c|}
\hline Regressão & 1 & 2 & 3 & 4 & 5 & 6 & 7 & 8 & 9 & 10 & 11 & 12 & 13 & 14 \\
\hline 1 & & 0,7750 & 0,0298 & 0,0629 & 0,7785 & 0,6374 & 0,2118 & 0,2363 & 0,1168 & 0,9952 & 0,4691 & 4,4581 & 0,8582 & 2,6664 \\
\hline 2 & & & 0,6456 & 0,9253 & 0,2075 & 0,9483 & 0,5739 & 0,1559 & 0,2914 & 0,1335 & 0,7785 & 2,3222 & 0,8187 & 2,1178 \\
\hline 3 & & & & 0,1740 & 0,7994 & 0,3980 & 0,0828 & 0,1829 & 0,0793 & 0,9632 & 0,4082 & 4,5747 & 1,0402 & 2,9863 \\
\hline 4 & & & & & 0,7005 & 1,0974 & 0,4931 & 0,3396 & 0,2229 & 0,9721 & 0,5460 & 4,0386 & 0,5720 & 2,0905 \\
\hline 5 & & & & & & 1,6764 & 0,9805 & 0,2578 & 0,3904 & 0,0332 & 0,1183 & 1,5518 & 0,2222 & 1,0185 \\
\hline 6 & & & & & & & 0,1285 & 0,6415 & 0,5459 & 1,6881 & 0,9124 & 6,2031 & 0,5438 & 0,7382 \\
\hline 7 & & & & & & & & 0,2329 & 0,1538 & 1,0578 & 0,4492 & 4,9349 & 1,4890 & 0,5823 \\
\hline 8 & & & & & & & & & 0,0223 & 0,3166 & 0,0448 & 3,0461 & 0,6194 & 2,1261 \\
\hline 9 & & & & & & & & & & 0,4915 & 0,1285 & 3,4968 & 0,6897 & 2,3251 \\
\hline 10 & & & & & & & & & & & 0,1288 & 1,4285 & 0,4121 & 1,2143 \\
\hline 11 & & & & & & & & & & & & 2,4076 & 0,5283 & 1,8059 \\
\hline 12 & & & & & & & & & & & & & 1,6783 & 0,8311 \\
\hline 13 & & & & & & & & & & & & & & 0,5021 \\
\hline \multicolumn{15}{|l|}{14} \\
\hline \multicolumn{15}{|c|}{ Início da formação do grupo 1} \\
\hline 8 e 9 & 0,1251 & 0,1565 & 0,0948 & 0,1949 & 0,2235 & 0,4032 & 0,1363 & - & - & 0,2768 & 0,0652 & 2,1884 & 0,4438 & 1,4912 \\
\hline \multicolumn{15}{|c|}{ Final da formação do grupo 1} \\
\hline $\begin{array}{l}1,2,3,4,5,6,7 \\
8,9,10,11 \text { e } 13\end{array}$ & - & - & - & - & - & - & - & - & - & - & - & 0,9738 & - & 0,8133 \\
\hline
\end{tabular}




\section{Conclusões}

1. O método de identidade de modelos pode ser usado com sucesso no agrupamento de equações de regressão linear simples, obtidas pelo método de Eberhart \& Russell (1966), para estudo de adaptabilidade e estabilidade de genótipos.

2. O método de agrupamento de modelos similares permite formar grupos de genótipos com comportamento estatisticamente semelhante.

\section{Referências}

CARVALHO, C.G.P. de; ARIAS, C.A.A.; TOLEDO, J.F.F. de; ALMEIDA, L.A. de; KIIHL, R.A. de S.; OLIVEIRA, M.F. de. Interação genótipo $\mathrm{x}$ ambiente no desempenho produtivo de soja no Paraná. Pesquisa Agropecuária Brasileira, v.37, p.989-1000, 2002

COSTA, J.G. da. Avaliação de compostos de milho (Zea mays L.) “dentado" provenientes de seleção recorrente recíproca. 1995. 102p. Dissertação (Mestrado) - Universidade Federal de Viçosa, Viçosa.

CRUZ, C.D. Programa GENES: biometria. Viçosa: UFV, 2006. $452 \mathrm{p}$.

CRUZ, C.D.; REGAZZI, A.J.; CARNEIRO, P.C.S. Modelos biométricos aplicados ao melhoramento genético. 2.ed. Viçosa: UFV, 2004. 480p.

EBERHART, S.A.; RUSSELL, W.A. Stability parameters for comparing varieties. Crop Science, v.6, p.36-40, 1966.

GOMES, F.P. Curso de estatística experimental. 13.ed. Piracicaba: Nobel, 1990. 468p.
GRAYBILL, F.A. Theory and application of the linear model. Belmont: Duxbury, 1976. 704p.

LEONEL, F. de P.; PEREIRA, J.C.; VIEIRA, R.A.M; FREITAS, J.A. de; DUTRA, A.R.; LIMA, A.V. de; RIBEIRO, M.D.; COSTA, M.G. Exigências nutricionais em macronutrientes minerais $(\mathrm{Ca}, \mathrm{P}$, $\mathrm{Mg}$, Na e K) para novilhos de diferentes grupos genéticos. Revista Brasileira de Zootecnia, v.35, p.584-590, 2006.

MAGALHÃES, S.R.S.; VEIGA, R.D.; SÁFADI, T.; CIRILLO, M.Â.; MAGINI, M. Avaliação de métodos para a comparação de modelos de regressão por simulação de dados. Semina: Ciências Exatas e Tecnológicas, v.25, p.117-122, 2004.

REGAZZI, A.J. Teste para verificar a identidade de modelos de regressão. Pesquisa Agropecuária Brasileira, v.31, p.1-17, 1996.

REGAZZI, A.J. Teste para verificar a identidade de modelos de regressão e a igualdade de alguns parâmetros num modelo polinomial ortogonal. Revista Ceres, v.40, p.176-195, 1993.

REGAZZI, A.J. Teste para verificar a identidade de modelos de regressão e a igualdade de parâmetros no caso de dados de delineamentos experimentais. Revista Ceres, v.46, p.383-409, 1999.

VÉRAS, A.S.C.; VALADARES FILHO, S. de C.; SILVA, J.F.C. da, PAULINO, M.F.; CECON, P.R., FERREIRA, M. de A.; LEÃO, M.I.; VALADARES, R.F.D.; MORAES, E.H.B.K. de. Predição da composição corporal e dos requisitos de energia e proteína para ganho de peso de bovinos, não cadastrados, alimentados com rações contendo diferentes níveis de concentrado. Revista Brasileira de Zootecnia, v.30, p.1127-1134, 2001.

ZERVOUDAKIS, J.T.; PAULINO, M.F.; DETMANN, E.; VALADARES FILHO, S. de C.; LANA, R. de P.; CECON, P.R.; QUEIROZ, D.S. de; MOREIRA, A.L. Conteúdo corporal e exigências líquidas de proteína e energia de novilhos suplementados no período das águas. Revista Brasileira de Zootecnia, v.31, p.530-537, 2002.

Recebido em 20 de agosto de 2010 e aprovado em 11 de novembro de 2010 